\title{
TPMT*V4 Allele
}

National Cancer Institute

\section{Source}

National Cancer Institute. TPMT*V4 Allele. NCI Thesaurus. Code C45797.

TPMT *V4 Allele is a variant form of 34-kb 10-exon human TPMT Gene (TPMT Family), which encodes polymorphic monomeric 245-aa 28-kDa cytoplasmic Thiopurine SMethyltransferase. Inhibited by S-adenosyl-L-homocysteine, TPMT catalyzes thiopurine S-methylation, an important metabolic pathway for thiopurine drugs such as 6mercaptopurine. TPMT contains a variable number of 17/18 bp tandem repeats (VNTR), from 3-9 (*V3-*V9), within the GC-rich 5'-flanking TPMT promoter region that modulate the level of TPMT enzyme activity (to a smaller extent than ORF-based SNP effects). A decrease in gene expression is seen with increasing repeat numbers. TPMT*V4 contains 4 VNTR. TPMT activity is thought to be an important determinant of toxicity associated with thiopurine medications. 